\title{
Comparison of on-wafer calibrations using the concept of reference impedance
}

\section{Francesc Purroy, Lluis Pradell}

Universitat Politècnica de Catalunya,

Department of signal theory and communications, ETSE

Telecomunicació. Ap. 30002 - 08080 Barcelona. Spain

\section{Abstract}

A novel method that allows to compare different calibration techniques has been developed. It is based on determining the reference impedance of a given Network Analyzer calibration from the reflection coefficient measurement of a physical open circuit. The method has been applied to several on-wafer calibrations.

\section{Introduction}

Many self-calibration techniques of Network Analyzers have been proposed to date. A consider effort has been also devoted to develop methods to compare calibrations. One way to do that comparison is measuring a known standard and define an error function that allow to compare different techniques. Another way consist of computing a calibration reference impedance for each technique, and compare the different values.

Some authors [1] assume the reference impedance of a calibration based on lines (TRL) to be the characteristic impedance of these lines, and the reference impedance of a calibration based on a match (LRM) to be the match impedance. However this assumption is not so obvious as it seems, and less obvious when a technique like LRM(known-reflect) is used. Other authors [2] obtain different results computing the reference impedance from a benchmark TRILL calibration.

In the line of the work presented last year [3] we propose a novel method, consistent with the reference impedance concept, without making any assumptions about the reference impedance nature. This method gives a direct way to compare different techniques because it does not refer the reference impedance to any benchmark calibration.

\section{Reference Impedance Concept}

We define the reference impedance associated to a given calibration procedure as the impedance to which the $S$ parameters keep actually referred after completing calibration. In consequence the measured (computed $\Gamma_{\text {comp }}$ ) reflection coefficient can be expressed in terms of a complex reference impedance [4] using the following expression:

$$
\Gamma_{\text {comp }}=\frac{Z_{\text {act }}-Z_{\text {ref.imp }}^{*}}{Z_{\text {act }}+Z_{\text {refimp }}}
$$

Where $\left(\mathrm{Z}_{\mathrm{act}}\right)$ is the actual value of the load under test, and $\left(\mathrm{Z}_{\text {ref.imp }}\right)$ is the reference impedance of the calibration. Although it is a widely used concept, it has not been yet shown that the calibration errors due to the incomplete knowledge of the calibration standards can be represented by a change in the reference impedance. Suppose the simple case of a one port OSL (Open-Short-Load) calibration performed using perfectly known and ideal open and short standards and a load standard not ideal whose reflection coefficient is not known $\left(\Gamma^{\mathrm{M}}{ }_{\text {act }}\right)$. Three error coefficients: a',b' and c' are obtained with some error: $\mathrm{E} a=\mathrm{a}-\mathrm{a}$ ', $E b=b-b^{\prime}$ and $E c=c-c^{\prime}$, where $a, b, c$ are the actual error coefficients ( $a=e$ loeol-eooe11, b=eoo, and $c=-e 11$ ). Suppose that $a=1, b=e 00$ and $c=0$. The errors due to the incorrect knowledge of the load reflection coefficient $\left(\mathrm{Ea}=1-\mathrm{a}\right.$ ', $\mathrm{Eb}=\mathrm{b}-\mathrm{b}$ ', $\left.\mathrm{Ec}=-\mathrm{c}^{\prime}\right)$ are related each other as follow:

$$
\begin{aligned}
& E b=-\Gamma^{M}{ }_{a c t} \\
& E a-E b=E c(b-1) \\
& E a+E b=E c(b+1)
\end{aligned}
$$

- Using (2) the computed reflection coefficient can be expressed as follows:

$$
\begin{gathered}
\Gamma_{\text {comp }}=\frac{Z_{a c t}-Z o \cdot \frac{1-E b}{1+E b}}{Z_{a c t}+Z o \cdot \frac{1-E b}{1+E b}} . \\
\Rightarrow \Gamma_{c o m p}=\frac{Z_{a c t}-Z^{M_{a c t}}}{Z_{a c t}+Z^{M_{a c t}}}
\end{gathered}
$$

Assuming that $\mathrm{Z}^{\mathrm{M}}$ act is real, this expression shows that the reference impedance is the impedance of the actual match. In addition it corrects the errors dues to inaccurate knowledge of the load calibration standard. Although the meaning of the reference impedance is not clear and should be investigated further, it can be used as a estimator of the goodness of a calibration method in order to compare different methods.

\section{Comparison Method}

The first step is to perform the different calibrations that will be compared. Then the calibrations are used to measure a known impedance. In order to have an optimum comparison the measured impedance should be the same for all calibrations. A good choice is a physical open circuit.

The open circuit can be modeled as a capacitor, with an unknown capacitance. For on-wafer calibration we propose to compute the capacitance value by enforcing the reference impedance of a LRM (Ideal Match) calibration at a given low frequency to be exactly $50 \Omega$. This is justified because the Match standard in a LRM (Ideal Match) calibration is a resistor trimmed to $50 \Omega$ within a $0.3 \%$ error. From this assumption and using (1) the reference impedance of any calibration can be computed from the next expressions:

$$
\begin{gathered}
\Re e\left(Z_{\text {ref.imp }}\right)=\frac{1}{C \cdot \omega} \cdot \Im m\left(\frac{1-\Gamma_{\text {comp }}}{1+\Gamma_{\text {comp }}}\right) \\
\Re m\left(Z_{\text {ref.imp }}\right)=-\frac{1}{C \cdot \omega} \cdot \Re e\left(\frac{1-\Gamma_{\text {comp }}}{1+\Gamma_{\text {comp }}}\right)
\end{gathered}
$$

- Where $\Gamma_{\text {comp }}$ is the computed reflexion coefficient of the known test impedance, and it is assumed that:

$$
\frac{Z_{\text {ref.imp }}^{*}}{Z_{\text {ref.imp }}} \approx 1
$$

\section{Experimental Results and Discussion}

In order to test this method we applied this technique to several on-wafer self-calibration methods, using a SUMMIT 9000 On-Wafer Station [5] provided with a LRM calibration substrate. The different calibration techniques are shown in the following table.

Note that when an on-wafer calibration is performed two different physical open circuits can be chosen: First reflect is 


\begin{tabular}{|c|c|c|c|c|}
\hline NAME: & THRU: & REFLECT: & $\begin{array}{l}\text { 3th } \\
\text { STANDARD: }\end{array}$ & SIMROL \\
\hline $\begin{array}{c}\text { L.RM } \\
\text { (KNOW-MATCH) }\end{array}$ & 1 ps LINE & AIR-OPEN/STUB-OPEN & MATCH & $\ldots$ \\
\hline LRRM & " & $\begin{array}{l}\text { AIR-OPEN,SHORT/ } \\
\text { STUB-OPEN,SHORT }\end{array}$ & MATCH & ..... \\
\hline TRL & $n$ & AIR-OPEN/STUB-OPEN & $\begin{array}{l}\text { 10\&40ps } \\
\text { LINE }\end{array}$ & $-\cdots$ \\
\hline TAR & $n$ & $\sim$ & $\begin{array}{c}10 \mathrm{~dB} \\
\text { ATTENUATO } \\
\mathrm{R}\end{array}$ & 00000 \\
\hline $\begin{array}{c}\text { LRM } \\
\text { (XNOW-REFLECT) }\end{array}$ & " & $n$ & MATCH & 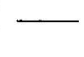 \\
\hline
\end{tabular}

done by leaving the probes in the air (referred to as air-open), second is done using a lps open-ended stub (referred to as stubopen). If a calibration using a stub-open instead of an air-open as the reflect standard is performed, the tip-to-substrate transitions are included in the error terms. In this case the air-open can not be modeled as a capacitor, because transition effects are not included in it. Therefore, a stub-open has to be used as the known test impedance in order to compute the reference impedance when a stub-open is used as a reflect in a known match LRM calibration.

However we tested both possibilities, which are shown in Fig 2 , and Fig 3 . In Fig 2 the computed reference impedance using a stub-open as a reflect, except for the LRM(Known-reflect) calibration where an air-open has been used as the reflect standard, has been plotted. In Fig 3, an air-open has been used as the reflect standard.

If the reference impedance of TRL, when a air-open has been used as a reflect Fig 3., is examined and compared with Fig 1, where the characteristic impedance of a $40 \mathrm{ps}$ line is plotted, a similar frequency behaviour is observed. On the other hand if the comparison is done between Fig 1 and Fig 3 the behaviour is more different. The low-frequency increment due to the loses effect can be minimised if we use a stub-open as reflect standard, because losses are included in all the standards and thus corrected by the calibration (Fig 2).
Another important point is that the 'overlap' effect [6] can be corrected simply by using a stub-open as the reflect standard. This can be seen in Fig. 1 where LRM (Known-Match) and LRRM calibrations, that has been developed to overcome the 'overlap' effect, give a very similar reference impedance value.

It is important to observe that the TAR calibration exhibit the worst reference reactance behaviour (Fig 2.) due to the fact that the attenuator impedance is much less ideal than the Match or Line standard impedances.

\section{Conclusions}

In this paper the reference impedance concept has been defined as well as an easy way to compute it under some hypothesis. This method has been used to compare different calibration techniques. It has been shown that the low-frequency behaviour of the transmission lines characteristic impedance can be corrected by the calibration if a stub-open is used instead of an air-open as the reflect standard. Another important result is that the 'overlap' effect is corrected by the calibration if a stub-open is used as the reflect standard giving a similar result to LRRM.

\section{References}

[1] F. Williams. "Characterization of Thin-Film Calibration Elements.". ARTFG Conference Digest, pp 25-35. Winter 1991.

[2] D.F. Williams, R.B. Marks. "Comparison of On-Wafer Calibrations". 38th ARTFG Conference Digest, pp 68-81. Winter 1991.

[3] Ll. Pradell, M. Cáceres, F. Purroy. "Development of SelfCalibration Techniques for On-Wafer and Fixtured measurements: A novel approach". 22th European Microwave Conference, pp 919924. Helsinki. Sept. 1992

[4] K. Kurokawa. "Power Waves and Scattering Matrix". IEEE Trans. Microwave Theory and Tech., vol MTT-13, pp 194-202, Mar 1965.

[5] CASCADE MICROTECH. "SUMMIT 9000. Analytical probe station. Instruction Manual"

[6] A. Davidson, K. Jones, E. Strid. "LRM and LRRM Calibrations with automatic determination of load inductance". 36th ARTFG Conference Digest, pp 57-63. November 1990.
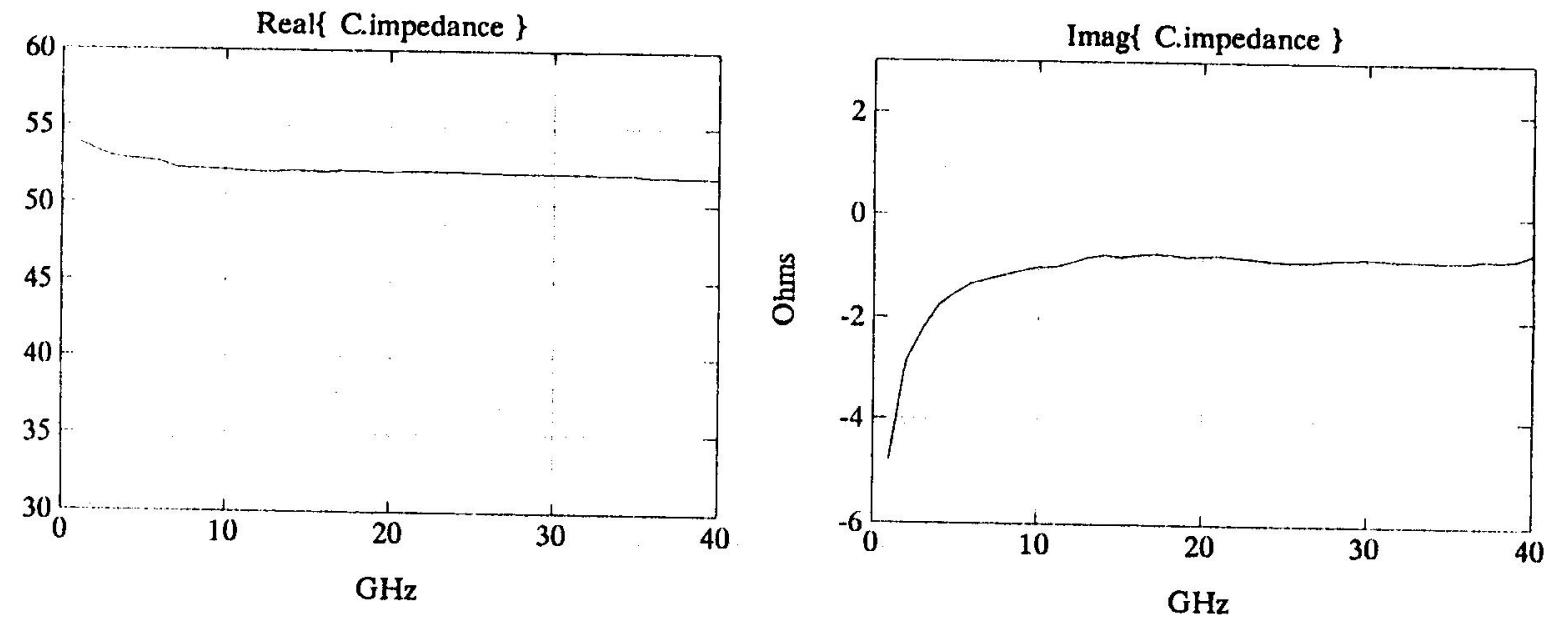

Fig 1.- Characteristic impedance of a CPW line. 

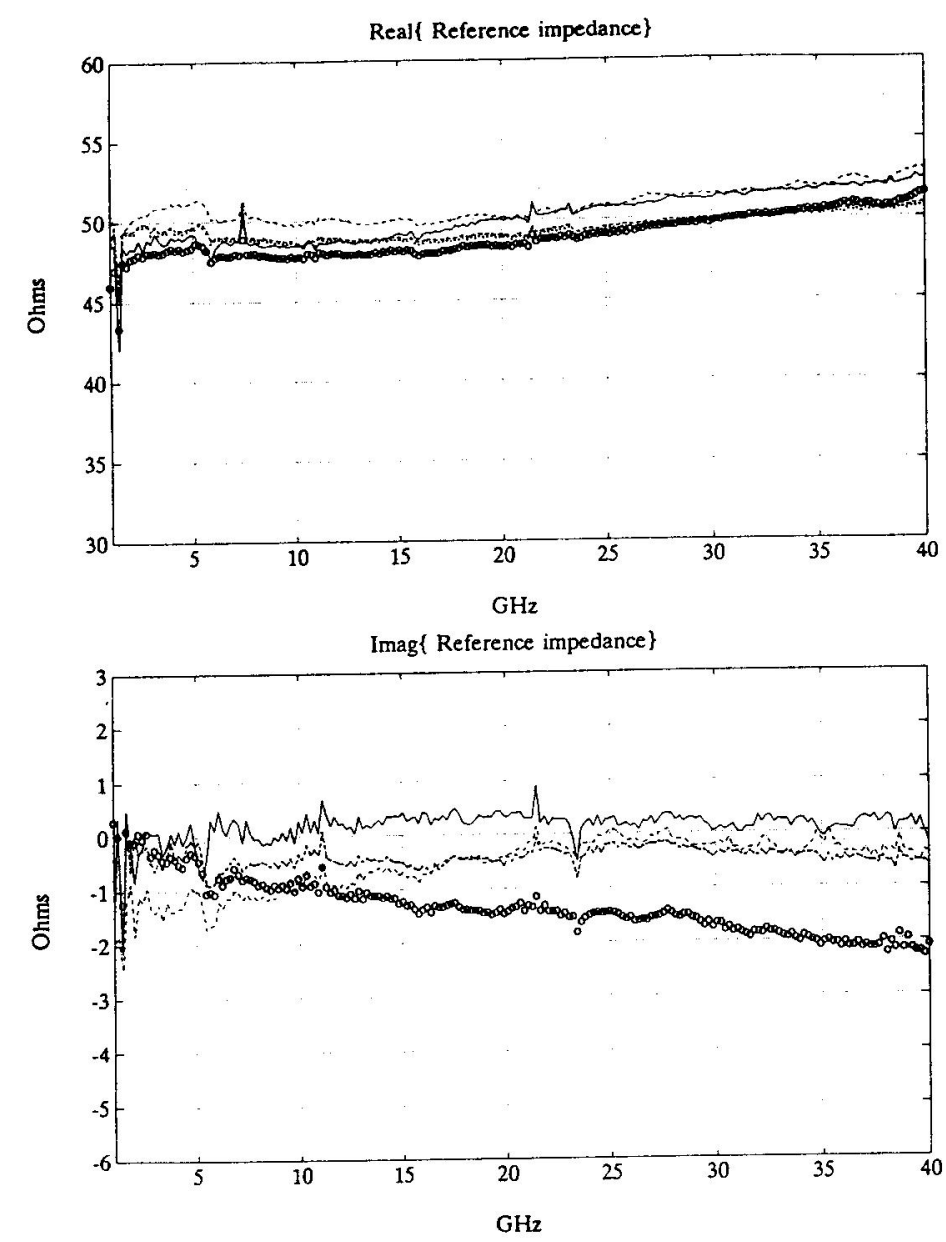

Fig.2. - Reflect $=$ stub-open, for all calibrations

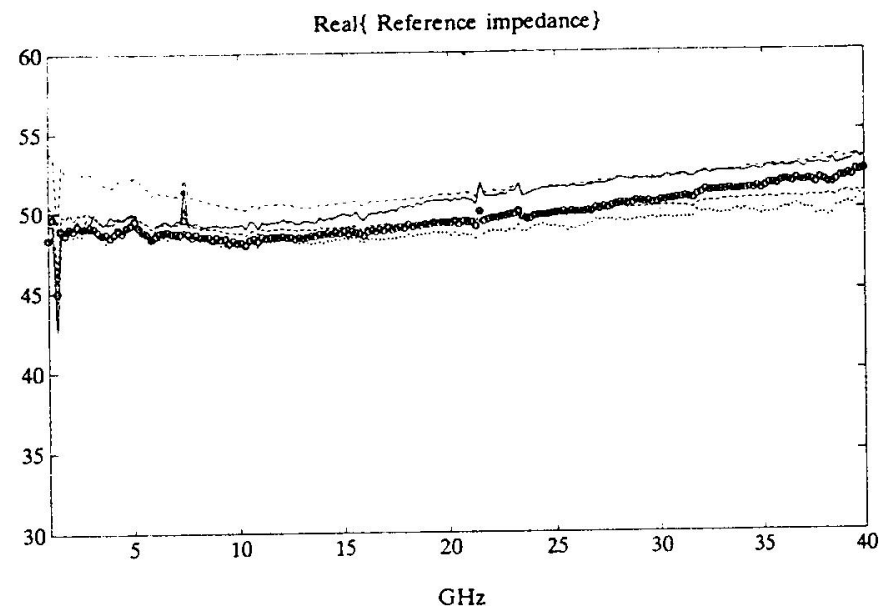

Imag $\{$ Reference impedance $\}$

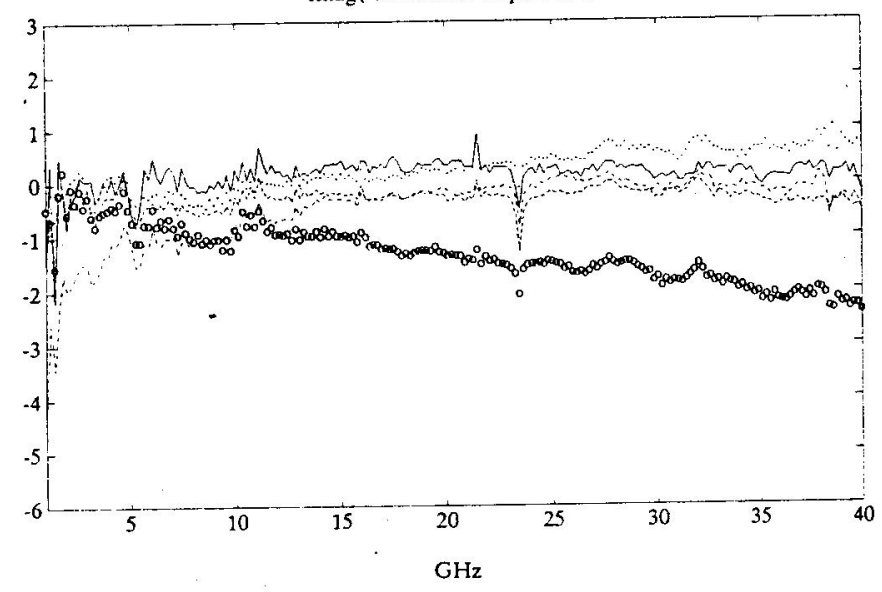

Fig. 3. - Reflect = air-open

except :LRM(Known-Reflect), Reflect = air-open

(*) LRM(Known-Match)[--],LRRM|-,TRL-.],TAR[oo],LRM(Known-Kentect)[-1 